\title{
Effects of rosmarinic acid on cognitive and biochemical alterations in ovariectomized rats treated with D-galactose
}

\author{
Deniz Kantar Gok ${ }^{1}$, Nihal Ozturk ${ }^{1}$, Hakan Er ${ }^{1}$, Mutay Aslan ${ }^{2}$, \\ Necdet Demir ${ }^{3}$, Narin Derin ${ }^{1}$, Aysel Agar ${ }^{4}$, Piraye Yargicoglu ${ }^{1}$
}

${ }^{1}$ Department of Biophysics, Akdeniz University, Faculty of Medicine, Arapsuyu, Antalya, Turkey

${ }^{2}$ Department of Biochemistry, Akdeniz University, Faculty of Medicine, Arapsuyu, Antalya, Turkey

${ }^{3}$ Department of Histology, Akdeniz University, Faculty of Medicine, Arapsuyu, Antalya, Turkey

${ }^{4}$ Department of Physiology, Akdeniz University, Faculty of Medicine, Arapsuyu, Antalya, Turkey

\begin{abstract}
Introduction. Animal models designed to mimic certain features of Alzheimer's disease (AD) can help us to increase our understanding of the underlying mechanisms of disease. Previous studies have revealed that long-term D-galactose injection combined with ovariectomy results in pathophysiologic alterations associated with AD. Thus, the aim of the present study was to investigate the effects of rosmarinic acid (RA) administration on pathological changes associated with ovariectomy and D-galactose injection, which serve as a two-insult model for AD.

Material and methods. One hundred female Wistar rats were divided into five equal groups: control (C), Sham (Sh), rosmarinic acid treated (R), ovariectomized rats treated with D-galactose (OD), ovariectomized rats treated with D-galactose and rosmarinic acid (ODR) groups. D-galactose $(80 \mathrm{mg} / \mathrm{kg} / \mathrm{day})$ was administered by i.p. injection and RA ( $50 \mathrm{mg} / \mathrm{kg} / \mathrm{day})$ was given via gavage for 60 days. Open field and Y-maze tests were used to assess locomotor activity and short-term spatial memory, respectively. Biochemical and histopathological analyses of the brain tissue were performed.

Results. Open field testing showed that the locomotor activity and exploratory behavior of rats were prominently impaired in the OD group as compared to the other studied groups. Similarly, Y-maze test results revealed a decrease of short-term spatial memory in the OD rats. A concomitant treatment with RA significantly restored altered locomotor activity and cognitive functions in the ODR group. Lipid peroxidation levels, cyclooxygenase-2 expression and prostaglandin E2 levels in the brain tissue were higher in the OD group and RA treatment inhibited these changes. $\mathrm{AD}$-like histopathological alterations and amyloid $\beta$ peptide $(\mathrm{A} \beta)$ depositions were observed in the OD group. Normal cell structure and lower $\mathrm{A} \beta$ depositions were observed in the ODR group compared with the OD group.

Conclusions. RA could have the potential to prevent some psychological and biochemical alterations of brain tissue found in a rat model of $\mathrm{AD}$ probably by attenuating lipid peroxidation and inflammatory response. (Folia Histochemica et Cytobiologica 2015, Vol. 53, No. 4, 283-293)
\end{abstract}

Key words: Alzheimer's disease; rat; estrogen deficiency; amyloid beta; rosmarinic acid; lipid peroxidation; COX-2; PGE2; short-term memory; hippocampus; electron microscopy; IHC

Correspondence address: Prof. Dr. P. Yargicoglu

Faculty of Medicine, Akdeniz University

Arapsuyu, 07070 Antalya

tel.: 0 090-242-2496906

fax: 0 090-242-2496903

e-mail: pakkiraz@akdeniz.edu.tr

\section{Introduction}

The deleterious consequences and pathophysiological role of increased reactive oxygen species (ROS) production are well known in Alzheimer's disease (AD) [1]. The early involvement of oxidative stress in $\mathrm{AD}$ is 
demonstrated by oxidative modifications of lipids $[2,3]$ and proteins $[4,5]$ in brains from $\mathrm{AD}$ patients, and also in animal models of AD. Additionally, overexpression of cyclooxygenase-2 (COX-2) and elevated levels of prostaglandin E2 (PGE2) [6, 7] have been observed in the AD. Since PGE2, a principal proinflammatory product of COX-2 enzymatic activity, is a potent inducer of inflammation [8,9], it could be expected that attenuating COX-2 and PGE2 signaling and free radical damage may represent good therapeutic targets against progresssion of $\mathrm{AD}$.

In a rat model of $\mathrm{AD}$ molecular alterations can be induced by long-term ovarian hormone deprivation combined with D-galactose (D-gal) treatment $[10,11]$. It is well known that estrogen deprivation $[12,13]$ and oxidative stress $[14,15]$ have been implicated as important risk factors in the pathogenesis of $\mathrm{AD}$. Estrogen deficiency after menopause has been shown to contribute to $\mathrm{AD}$ development and considering the reported neuroprotective effects of estrogens [16], estrogen replacement therapy (ERT) has been suggested to be useful for preventing the onset of this disease. However, considering the well-defined side effect of ERT $[17,18]$, the use of estrogen as a treatment for AD is limited. Hence, there is a need to develop alternative $\mathrm{AD}$ therapeutic agents with fewer side effects.

Members of the Salvia species, such as Salvia officinalis and Salvia lavandulaefolia, have a long history of usage as traditional herbal medicine in many cultures. Salvia extract is generally known for its multiple biological activities including anticholinergic, anti-inflammatory and antioxidant properties potentially relevant to brain function [19, 20]. In previous clinical studies, it was shown that Salvia extracts improved cognitive performance and enhanced positive mood in young healthy volunteers [21-23]. In addition, a Salvia extract improved memory and attention in healthy older subjects by its cholinesterase inhibiting properties by inhibiting cholinesterase activities [24]. Beside healthy individuals, a randomized double-blind clinical study showed that an ethanolic extract from Salvia officinalis which contains rosmarinic acid (RA) was effective in the management of mild to moderate $\mathrm{AD}[25,26]$. Rosmarinic acid is a naturally occurring phenolic compound that is known for its antioxidant, anti-cholinergic and anti-inflammatory activities [27-30]. So, the aim of the present study was to investigate possible protective effect of RA on symptoms seen in the $\mathrm{AD}$ rat model by behavioral, neurochemical and histopathological analyses.

\section{Material and methods}

Animals. The study protocol was approved by Akdeniz University Animal Care and Use Committee. A hundred healthy female albino Wistar rats, aged five months, weighing 300 to $350 \mathrm{~g}$ were used. Animals were housed in stainless steel cages in groups of 4 rats per cage at standard conditions $\left(24 \pm 2^{\circ} \mathrm{C}\right.$ and $50 \pm 5 \%$ humidity) with $12 \mathrm{~h}$ light-dark cycle and given food and water ad libitum. The experiments were performed between 9:00 and 17:00. Rats were randomly divided into five groups ( $\mathrm{n}=20$ per group): Group 1: control (C); Group 2: rats were sham operated and treated with saline $(\mathrm{SH})$; Group 3: rats were treated with rosmarinic acid (R); Group 4: rats were ovariectomized and treated with D-gal (OD; AD model); Group 5: rats were ovariectomized and treated concomitantly with D-gal and rosmarinic acid (ODR). D-galactose (Sigma-Aldrich, St. Louis, MO, USA; $80 \mathrm{mg} / \mathrm{kg} / \mathrm{day}$, $80 \mathrm{mg}$ of D-gal dissolved in $0.9 \%$ saline solution, to a total volume of $1 \mathrm{~mL}$ ) was administered by i.p. injection and RA (Carbosynth, San Diego, CA, USA; $50 \mathrm{mg} / \mathrm{kg} / \mathrm{day}, 50 \mathrm{mg}$ of RA dissolved in $0.9 \%$ saline solution, to a total volume of $1 \mathrm{~mL}$ ) was given via gavage for 60 days. Physiologic saline was administered by i.p. injection and gavage to the SH group for the same period of time.

Tissue preparation. After behavioral tests, rats were deeply anesthetized with urethane and brains of rats were perfused transcardially with heparinized saline, removed immediately and stored at $-80^{\circ} \mathrm{C}$ for biochemical analysis. For immunohistochemical studies, after transcardial perfusion with cold $0.9 \%$ saline followed by $4 \%$ paraformaldehyde (for immunohistochemistry, $\mathrm{n}=5$ ) or with $0.5 \%$ glutaraldehyde (for electron microscopy, $\mathrm{n}=3$ ) in phosphate-buffered saline (PBS, 0.01 M, $\mathrm{pH} 7.4$ ), the brains were removed and post-fixed overnight or $3 \mathrm{~h}$ at $4^{\circ} \mathrm{C}$ in the same solutions, respectively.

Immunohistochemistry. For anti-A $\beta$ antibody immunohistochemistry, sections were deparaffinized and blocked for endogenous peroxidase activity and nonspecific binding (BioGenex, San Ramon, CA, USA). Rabbit anti-A $\beta$ 40/42 antibody (1:200; Cell Signaling, Saint Louis, MO, USA) was applied for $1 \mathrm{~h}$ at room temperature. For negative control the primary antibody was replaced by normal rabbit IgG serum (Vector Laboratories, Burlingame, CA, USA) at the same concentration. After several washes, sections were incubated with biotinylated goat anti-rabbit IgG secondary antibody (1:400 dilution, Vector Lab.) followed by LSAB streptavidin-peroxidase complex (Dako, Carpinteria, CA, USA) incubation and were rinsed with PBS. Antibody complex was visualized by incubation with diaminobenzidine chromogen (BioGenex). Sections were counterstained with Mayer's hematoxylin (Dako), dehydrated, mounted and examined on a Zeiss-Axioplan, microscope (Zeiss, Oberkochen, Germany). A $\beta$ levels were quantified using Image J $(1.48 \mathrm{v})$ software (NIH, USA), which confers semi-quantitatively analysis of immunohistochemical staining.

Transmission electron microscopy. Samples of hippocampus were dissected from brain and fixed with $4 \%$ glutaraldehyde, 
prepared in $0.1 \mathrm{M}$ Sorensens's phosphate buffer solution (pH 7.4). Then, samples were postfixed with $1 \%$ osmium tetraoxide in the same buffered solution. After dehydration through a graded series of ethanol, they were embedded in epoxy resin (Araldite CY212, Agar Scientific Ltd, Stansted, UK). Semi-thin $(1 \mathrm{~mm})$ and ultrathin $(40-60 \mathrm{~nm})$ sections were cut with an ultramicrotome (Leica ultracut, UCT, Leica MZ6, England). Semi-thin sections $(1 \mu \mathrm{m})$ were stained with toluidine blue and were examined with a light microscope (Olympus CX41, Tokyo, Japan) for orientation. Afterwards, ultrathin sections (40-60 nm) were contrasted with uranyl acetate and Reynold's lead nitrate and were examined with a transmission electron microscope (LEO 906E model, Zeiss) [31]. Ultrastructural analysis was performed on the CA1 granular layer of the hippocampus to mainly focus on the morphologic changes of the nucleus, cytoplasmic organelles. For analysis of morphologic changes of organelles in the CA1, neurons in this layer were randomly selected and examined at $\times 6000$ magnification.

Determination of thiobarbituric acid reactive substances (TBARS). Levels of TBARS were measured by a fluorimetric method described by Wasowicz et al. [32], using 1,1,3,3-tetraethoxypropane as a standard. Brain tissues $(n=10$ per group) were weighed and homogenized (Bio-Gen Pro-200) in an ice cold $50 \mathrm{mM}$ potassium phosphate buffer at $\mathrm{pH}$ 7.0. Homogenates were centrifuged $\left(10.000 \mathrm{~g}, 15 \mathrm{~min}, 4^{\circ} \mathrm{C}\right)$ (3-18K centrifuge, Sigma-Aldrich) and supernatants were used for lipid peroxidation analysis. All measurements were performed in duplicate and assay was repeated three times. The results are given as nmol/g protein.

Western Blot analysis of 4-hydroxy-2-nonenal (4-HNE) and COX-2 proteins. Protein concentrations in all samples were measured spectrophotometrically by a protein assay reagent kit (Pierce, Rockford, IL, USA) via a modified Bradford method. Bovine serum albumin was used as standard. Western blot analysis was performed as previously described [33]. A rabbit polyclonal COX-2 antibody (1:200 dilution; Santa Cruz Biotechnology, Santa Cruz, CA, USA) and a rabbit polyclonal 4-HNE antibody (1:1000 dilution) were used for Western blot (WB) analysis. Horseradish peroxidase-conjugated goat anti-rabbit IgG (1:10000 dilution; Zymed Laboratories, San Francisco, CA, USA) was used as a secondary antibody and immunoreactive proteins were visualized on high performance chemiluminescence film via ECL (enhanced chemiluminescence) reagent (Amersham Pharmacia Biotech, Buckinghamshire, UK). Western blotting was performed two times. Densitometric analysis of immunoblots was performed using NIH image 1.61 software for windows (Scion Corporation, Frederick, MD, USA).

Measurement of PGE2 content in hippocampus. For determination of PGE2, isolated hippocampus tissues $(n=10$ per group) were placed in Tris-HCl buffer ( $\mathrm{pH} 7.5$ ) with $0.02 \mathrm{M}$ EDTA and $5 \mathrm{mg} / \mathrm{mL}$ indomethacin. PGE2 collection and purification was done according to a modified protocol supplied with the PGE2 EIA system (Cayman Chemical Company, Ann Arbor, MI, USA). The quantity of PGE2 was measure by enzyme immunoassay (EIA) according to the protocol provided with the EIA kit (Cayman). The assay was performed in duplicate and repeated twice.

Locomotor activity test. At the end of the 60 days experimental period, locomotion and exploratory behavior of rats were observed in a brightly illuminated square-shaped arena $(50 \mathrm{~cm} \times 50 \mathrm{~cm})$. The animal was placed in the center and allowed to explore the open field for $5 \mathrm{~min}$. The locomotor activity (path-length, resting time) was assessed using an automated tracking system (MAY ACT508, animal locomotor activity meter, Commat Ltd., Ankara, Turkey). After each test the arena was cleaned with $70 \%$ ethanol.

Short-term memory assessment by Y-maze test. After a 2-day rest period following locomotor activity test, short-term spatial memory was assessed using the Y-maze [34], which consisted of three arms made of black Plexiglas. The stem arm and the two arms forming the $\mathrm{Y}$ were 42 and $37 \mathrm{~cm}$ long, respectively, and diverged at a $45^{\circ}$ angle from the stem arm. The three arms were randomly designated: first arm - start arm, in which the rat started to explore (always open); second arm - novel arm, which was blocked at the $1^{\text {st }}$ trial, but open at the $2^{\text {nd }}$ trial; third arm — the other arm (always open). In the first phase of the experiment the rat was placed at the end of the start arm. During training, novel arm was blocked and each rat was allowed to explore the start and other arms for $10 \mathrm{~min}$. After a 2 hour interval, the rat was replaced in the Y-maze for $5 \mathrm{~min}$ and all arms were accessible. Rats were interpreted to have intact spatial memory if they showed a preference for the novel arm since rats have an innate tendency to explore novelty. Behavior was recorded on video and the number of times each arm was visited and the time spent in each arm was analyzed using video tracking (Ethovision XT, Noldus, The Netherlands).

Statistical analysis. The statistical analyses of the obtained data were performed by SPSS (version 18.0) software for windows. While results of Western blot were expressed as means \pm standard deviation, other results were expressed as means \pm standard errors. TBARS and locomotor activity values were analyzed via one-way analysis of variance (ANOVA) and all pairwise multiple comparisons were performed by Tukey's test. The differences in other parameters were analyzed via Kruskal-Wallis one-way analysis of variance on ranks, and all pairwise multiple comparisons were performed by Mann-Whitney U. Additionally, statistical analysis within each group for Y-maze data was performed by Wilcoxon paired t-test. $P$ values less than 0.05 were considered significant. 


\section{Results}

\section{Immunohistochemistry of amyloid beta expression}

There was a very little intraneuronal $\mathrm{A} \beta$ immunolabeling localized in hippocampal areas of $\mathrm{C}$ and $\mathrm{R}$ groups (Figure 1A: $\mathrm{C}, \mathrm{R}$ ). On the other hand, intense $\mathrm{A} \beta$ immunoreactivity was clearly observed in the cytoplasm of hippocampal neurons in the OD group (Figure 1A: OD). Moreover, there was a moderate $\mathrm{A} \beta$ immunolabeling detected in the hippocampus of ODR group (Figure 1A: ODR). A $\beta$ immunostaining in the ODR group was not as intense as in the OD group. Quantitative analysis of $\mathrm{A} \beta$ stained sections revealed higher immunoreactivity in the OD group versus the other groups and percentage of stained area decreased in the ODR group versus the OD group (Figure 1B).

\section{Ultrastructural analysis}

The electron microscopic analysis of the brains of control (C) rats revealed that the morphology of pyramidal neuron's nucleus $(\mathrm{N})$ and perikaryon $(\mathrm{P})$ was normal (Figure 2A). Additionally, cytoplasm and structure of organelles seemed also normal with maintained mitochondrial membranes structure in this group (Figure 2A). In the R group, normal cytoplasm, organelle structure together with regular mitochondrial (black arrows) and normal Golgi morphology (star) were viewed (Figure 2B). In ultrastructural analysis of OD group, typical pathological changes associated with neurodegeneration were detected in CA1 area of hippocampus. In this group, cytoplasmolysis (stars), edematous endoplasmic reticulum, severe crista deformations and mitochondrial swelling (black arrows) were observed in perikaryon. Besides these changes, wide spread membrane deformation (white arrows) and edema in nucleus (N) were also detected (Figure 2C). In the ODR group maintained mitochondrial membrane structure (arrowheads) drew attention in cytoplasm and neuropil of CA1 pyramidal cell. Nucleus $(\mathrm{N})$ membrane integrity was not impaired and normal perinuclear space (white arrows) was found (Figure 2D). As seen in Figure 2D, axons of hippocampal CA1 pyramidal cell showed normal structure and did not contain electron dense deposits (black arrows).

\section{Tissue lipid peroxidation}

Brain TBARS levels in OD group were significantly higher than those detected in other groups. Rosmarinic acid significantly decreased TBARS levels in the ODR group compared with the OD group. TBARS levels were significantly increased in the SH group versus $\mathrm{C}$ group. Rosmarinic acid treatment alone had no impact on lipid peroxidation in the $\mathrm{R}$ group with measured TBARS levels (Table 1).

\section{Levels of 4-HNE and COX-2 proteins as measured by Western blotting}

4-hydroxy-2-nonenal (Figure 3A, B) and COX-2 (Figure 3C, D) western blots and densitometric analysis of hippocampal tissues from all groups are shown in Figure 3. COX-2 protein levels and 4-HNE levels were increased in the OD group compared with the others. Decreased COX-2 levels were observed in the ODR group, compared with the OD group. A decrease in 4-HNE levels was detected in the hippocampus of the OD group treated with RA. Protein staining of brain homogenates applied for WB analysis shows equal protein loading in each lane (Figure 3E).

\section{Hippocampal PGE2 content}

As seen in Figure 4, PGE2 levels were significantly higher in the OD group compared with all other groups including ODR. Rosmarinic acid decreased PGE2 levels in the ODR group compared with the OD group. However, PGE2 levels were significantly $(\mathrm{p}<0.05)$ increased in ODR group compared with $\mathrm{C}, \mathrm{SH}$, and $\mathrm{R}$.

\section{Locomotor activity test}

There was a significant decrease in the distance moved by the OD group $(\mathrm{p}<0.001)$ compared to other groups. SH and ODR groups showed no significant change compared to the $\mathrm{C}$ group (Table 2). Distance moved by the rats of the $\mathrm{R}$ group was significantly higher than by rats of other groups.

A significant decrease in the time of resting was seen in the R group $(p<0.001)$ compared with the other groups (Table 2). The OD group showed significantly higher resting time when compared with other groups, ODR and SH groups showed no significant change compared to the $\mathrm{C}$ group.

\section{Short memory test (Y-maze test)}

The time spent (Figure 5A) and the number of visits (Figure 5B) to the novel arm compared with the familiar "other" arm were significantly lower in the OD group versus the other groups. In all groups except $\mathrm{OD}$, the time spent in the novel arm was significantly higher than those in the other arm. The number of visits to the novel arm versus the "other" arm were significantly higher in all groups except OD. Rosmarinic acid increased the time spent and the number of visits in the novel arm versus the "other" arm in the ODR group with respect to the OD group. There were no significant differences between groups for total arm entries, indicating that the deficit observed did not 


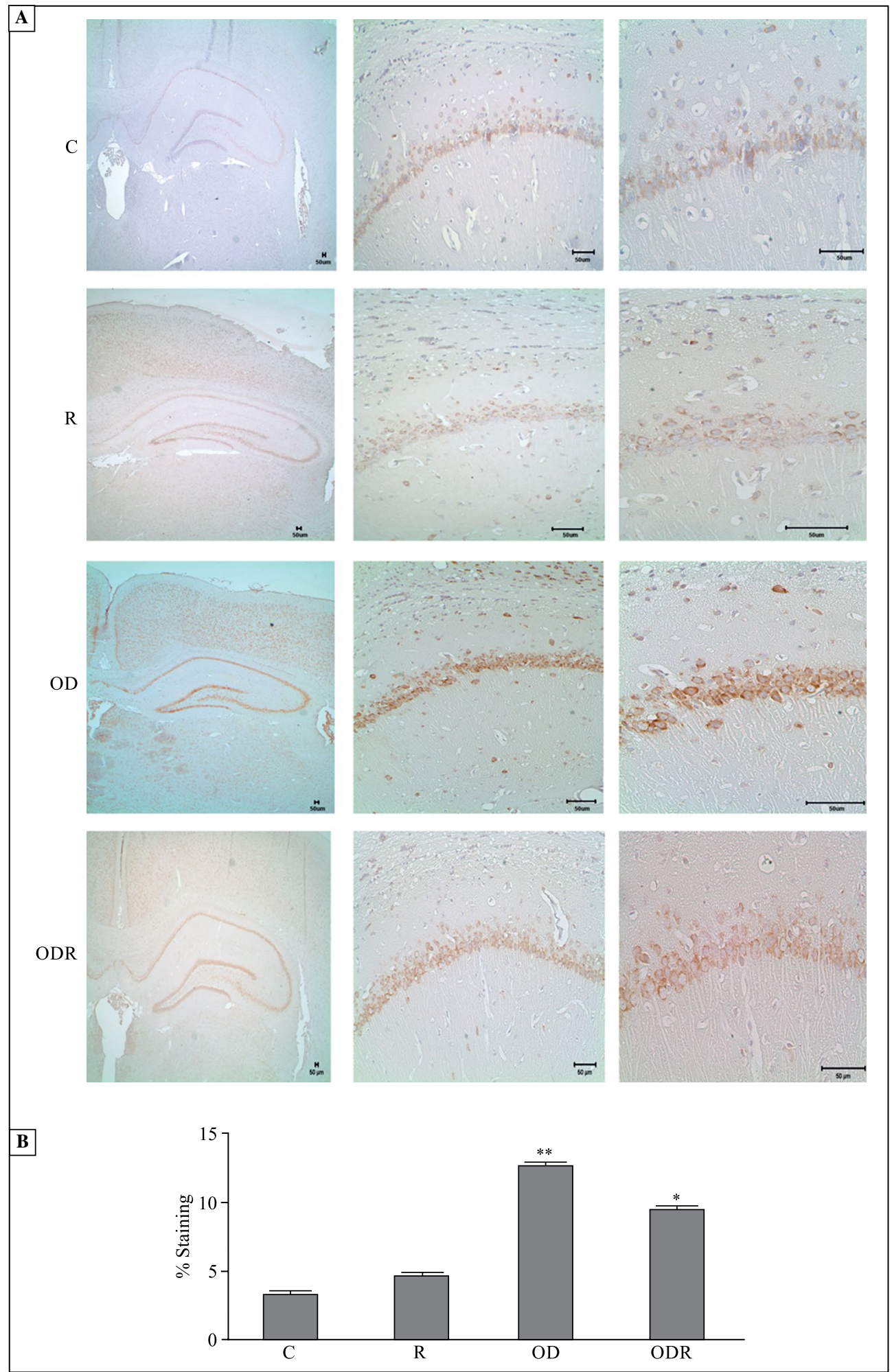

Figure 1. Immunohistochemical staining of amyloid $\beta$ protein in all experimental groups. A. Representative images of amyloid $\beta(\mathrm{A} \beta)$ peptide localization in the hippocampus. Middle and right panels show CA1 region. The magnifications are $\times 2.5, \times 20$ and $\times 40$ in left, middle and right panel, respectively; B. Quantitative analysis of A $\beta$ staining levels were compared in all groups, using ImageJ software. Results are mean \pm SEM, $n=5$ in each group. ${ }^{*} \mathrm{p}<0.05$; difference of ODR group versus the other groups, ${ }^{* *} \mathrm{p}<0.05$; difference of OD group versus the other groups. Abbreviations: $\mathrm{C}-\mathrm{control}$; $\mathrm{R}$ - rosmarinic acid (RA) treated; OD — ovariectomized rats treated with D-galactose (D-gal); ODR — ovariectomized rats treated with D-gal and RA 

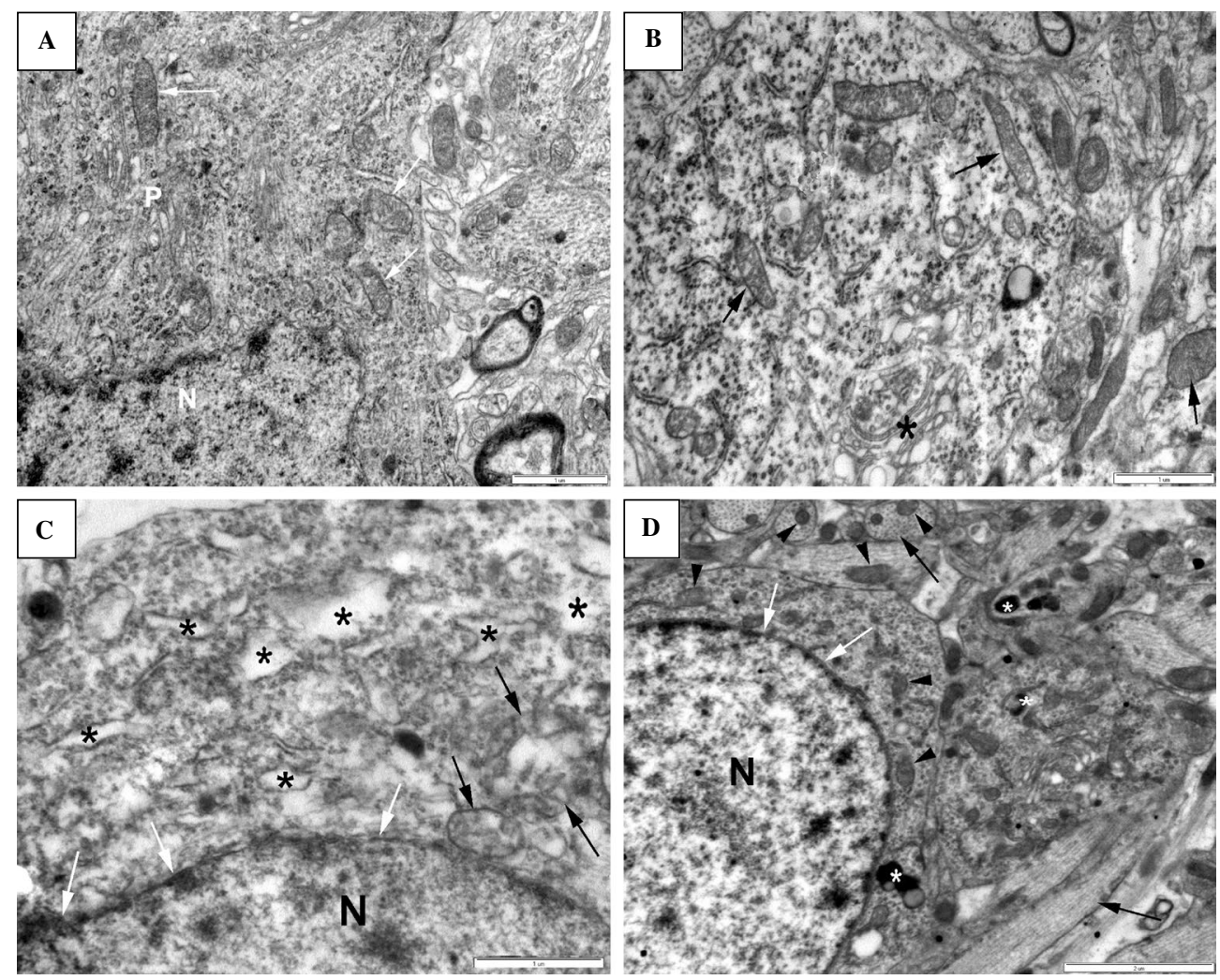

Figure 2. Representative ultrastructural images of CA1 of hippocampal tissue sections from each experimental group. A. The neuron nucleus $(\mathrm{N})$ and the perikaryon structure $(\mathrm{P})$ of the control group. Scale: $1 \mu \mathrm{m}$; B. Mitochondrias (black arrows) and Golgi (star), membrane structure of the R group. Scale: $1 \mu \mathrm{m}$; C. Nucleus membrane (white arrows), mitochondrias (black arrows) and cytoplasmolysis (stars) of the OD group. Scale: $1 \mu \mathrm{m}$; D. Nucleus (N), perinuclear space (white arrows), mitochondrial membrane (arrowheads) and axons (black arrows) of the ODR group. Scale: $2 \mu \mathrm{m}$. $\mathrm{n}=3$ in each group. Abbreviations as in the description of Figure 1

Table 1. Tissue lipid peroxidation expressed by the levels of TBARS

\begin{tabular}{|l|c|}
\hline Group & nmol/g protein \\
\hline C & $0.17 \pm 0.07$ \\
\hline SH & $0.28 \pm 0.11^{*}$ \\
\hline R & $0.17 \pm 0.08$ \\
\hline OD & $0.40 \pm 0.13^{* *}$ \\
\hline ODR & $0.20 \pm 0.07^{* * *}$ \\
\hline
\end{tabular}

The content of thiobarbituric acid reactive substances (TBARS) was measured as described in Material and methods. The results are presented as mean \pm SEM, $\mathrm{n}=10$ for each group. *Significantly different versus the $\mathrm{C}, \mathrm{R}$ and $\mathrm{OD}$ groups, $\mathrm{p}<0.05 ;{ }^{*}$ Significantly different versus the other groups, $\mathrm{p}<0.05 ; * * * \mathrm{p}<0.05$; significant difference between the ODR group versus the OD group

arise as a consequence of reduced activity in the ODR group (data not shown).

\section{Discussion}

It is known that estrogen has neurotropic and protective effects in the brain. In agreement with the knowledge that $\mathrm{AD}$ is more common in women [35], recent studies showed that decreased estrogen level is a risk factor for $\mathrm{AD}$ and estrogen deficiency has been implicated in $\mathrm{AD}$ etiology [36, 37]. Beside hormonal changes, increased oxidative stress with aging is another important risk factor for developing $\mathrm{AD}$. The long-term injection of D-gal contributes to the oxidative modifications in cerebral tissue, and further leads to lipofuscin deposition, slight neuronal damage and memory decay which are all prominent and early changes in aging [38]. A previous study has demonstrated that these two factors have synergistic effects on accelerating the progression of $\mathrm{AD}$ and long-term administration of D-gal $(80 \mathrm{mg} / \mathrm{kg} /$ day $)$ into 


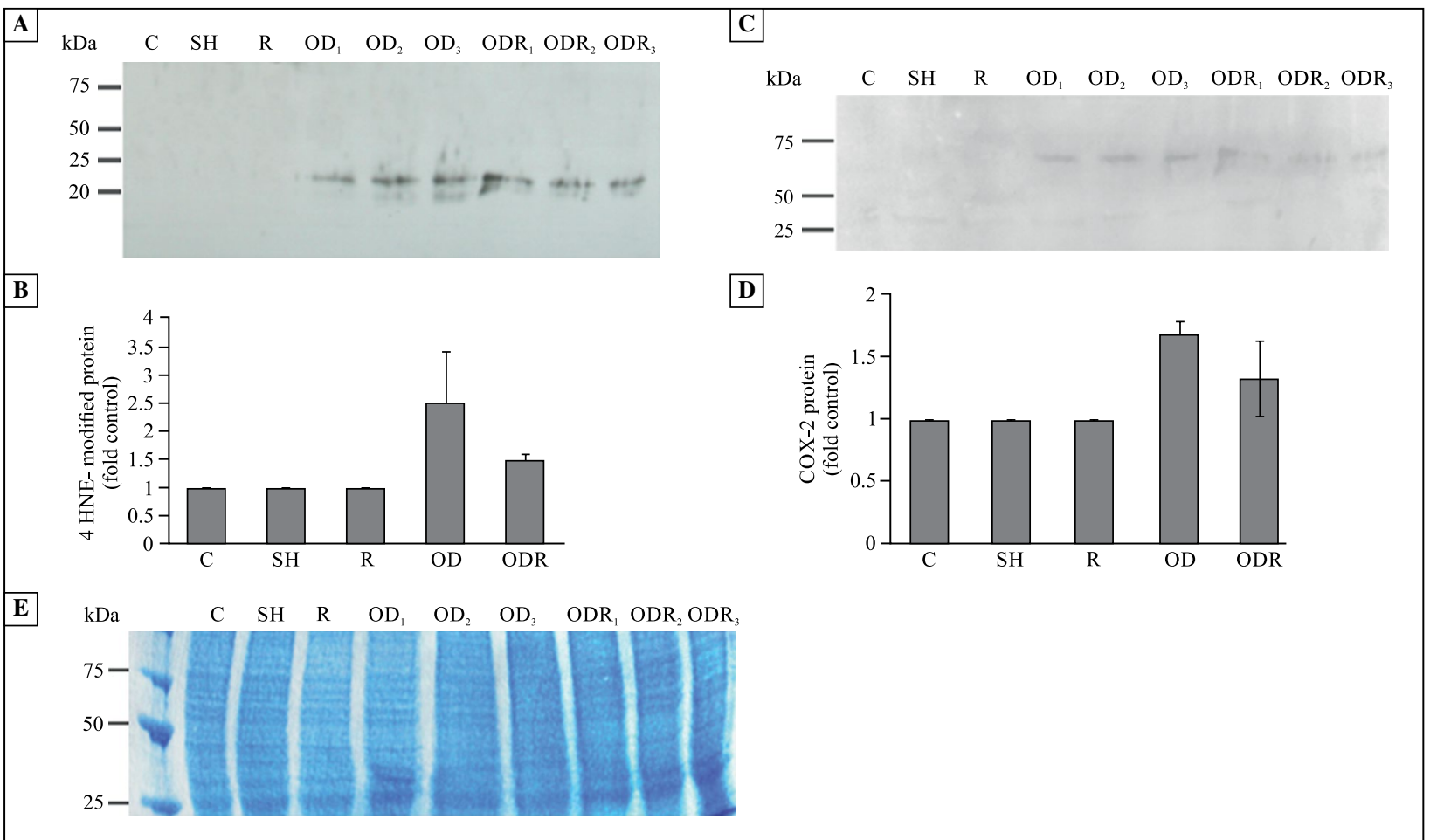

Figure 3. Western blotting (WB) analysis. A. WB of 4-HNE proteins; B. Densitometric analysis of 4-HNE proteins; C. WB of COX-2 protein; D. Densitometric analysis of COX-2 protein; E. SDS-PAGE analysis of hippocampal homogenates. Brain proteins were separated by SDS-PAGE and visualized by Coomassie blue staining. Results are presented as mean of two experiments. Error bars represent the standard deviation of duplicate experiments. $\mathrm{n}=1-3$ per experiment. Abbreviations as in the description of Figure 1

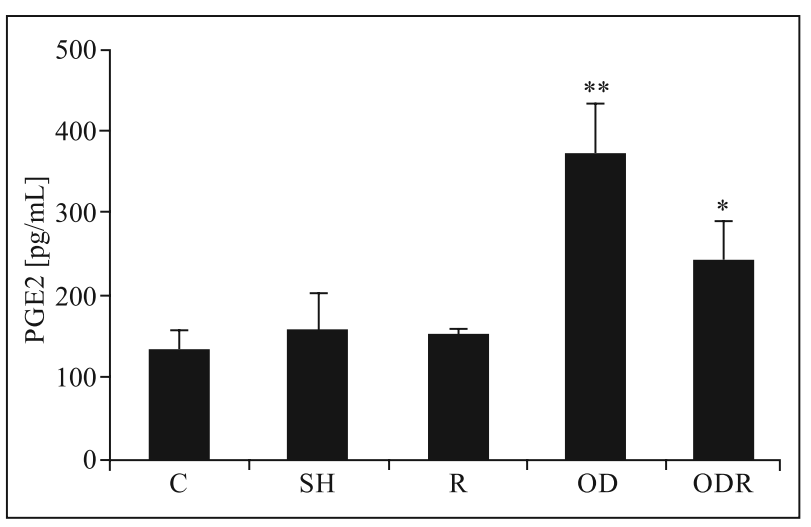

Figure 4. PGE2 levels in the hippocampus of control and experimental rats. The results are presented as mean \pm SEM, $\mathrm{n}=10$. *Significant difference between the ODR and other groups, $\mathrm{p}<0.05 ; * *$ Significant difference between the OD and other groups, $\mathrm{p}<0.05$. Abbreviations as in the description of Figure 1

ovariectomized rats may serve as an $\mathrm{AD}$ rodent model which can mimic the pathological, neurochemical and behavioral alterations observed in $\mathrm{AD}[10]$. Amyloid $\beta$ peptide deposition was examined in the hippocampus to confirm that the animal model can mimic the neuropathological manifestations observed in the disease.
Table 2. Locomotor activity test

\begin{tabular}{|l|c|c|}
\hline Group & Distance moved $[\mathrm{cm}]$ & Resting (\%) \\
\hline $\mathrm{C}$ & $531.9 \pm 61.03$ & $51.5 \pm 2.67$ \\
\hline $\mathrm{SH}$ & $556.8 \pm 97.8$ & $53.1 \pm 6.0$ \\
\hline $\mathrm{R}$ & $825.1 \pm 96.08^{*}$ & $39.9 \pm 7.51^{*}$ \\
\hline OD & $198.4 \pm 25.9^{* *}$ & $72 \pm 10.6^{* *}$ \\
\hline ODR & $538.2 \pm 96.2$ & $53 \pm 5.83$ \\
\hline
\end{tabular}

The locomotor activity test was performed as described in Material and methods. The results are presented as mean \pm SEM, $n=20$ for each group. *Statistically different versus the other groups, $\mathrm{p}<0.001$; **Statistically different versus the other groups, $\mathrm{p}<0.001$

Our findings support previously reported observations of $\mathrm{A} \beta$ immunoreactive neurons which were detected in the hippocampus of all ovariectomized rats treated with D-gal [10]. Also, in line with the previous study of Zhao et al. [39], we detected marked increased in lipid peroxidation levels in the OD rats. Our results and previously reported findings suggest that endogenous $\mathrm{A} \beta$ peptides aggregate to form amyloid plaques in the presence of oxidative and neurodegenerative milieu created by $\mathrm{D}$-gal treatment and estrogen deprivation. Consistent with the $\mathrm{A} \beta$ deposition, ultrastructural analysis displayed some pathological alterations 


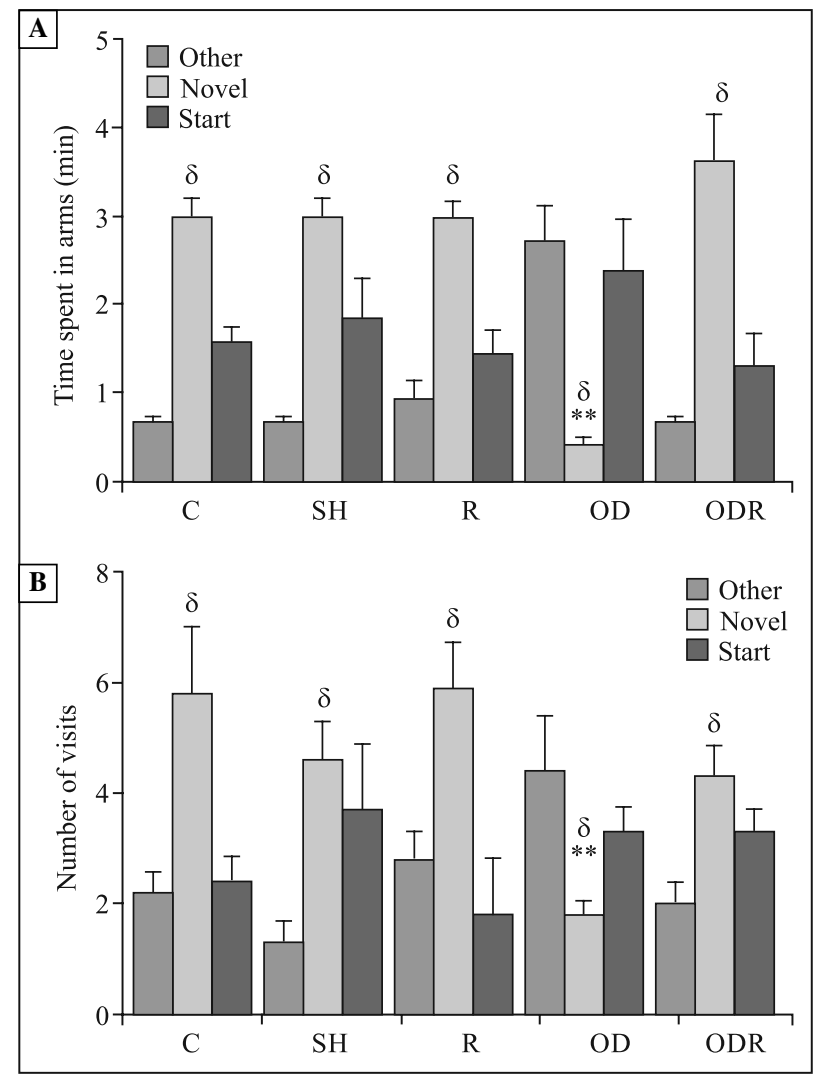

Figure 5. Y-maze test results in all groups. A. The time spent ( $\mathrm{min}$ ); B. Number of visits. The results are presented as mean \pm SEM, $n=20$ for each group. $\delta-$ significant difference between novel arm score versus other arm score in all groups, $\mathrm{p}<0.05 ; *$ Significant difference between novel arm score of the OD group versus the other groups, $p<0.05$. Abbreviations as in the description of Figure 1

such as edematous endoplasmic reticulum, severe mitochondrial deformations, nucleus membrane deformation and edema in OD group. These changes were in line with the earlier report revealing degenerations in nucleus and organelle structures in D-gal administered ovariectomized rats [10]. These data are in line with the previous reports which indicate that decreased estrogen levels after menopause and increased oxidative stress are risk factors for development and progression of the disease.

Although a large amount of evidence indicates that estrogen plays a neuroprotective role in $\operatorname{AD}[13,40]$, ERT is a controversial issue and has been linked to increase the risk of certain cancers, like breast and uterine cancer [17, 18]. Therefore, RA, which is a safe compound, was used in our study as therapeutic agent to attenuate the changes associated with AD. The therapeutic efficacy of RA has been tested in both in vitro and in vivo models of $\mathrm{AD}$. Previous studies have been tested the effect of RA on $\mathrm{A} \beta$ aggregation in both in vitro and transgenic mice model of $\mathrm{AD}$ $[41,42]$. It has been reported that RA prevents $A \beta$ oligomerization under in vitro conditions and that RA prevents $\mathrm{A} \beta$-induced alterations of neuronal plasticity in the CA1 region of mouse hippocampus slices [41]. Oral administration of RA decreased the $\mathrm{A} \beta$ burden in the $\mathrm{AD}$ model transgenic mice by inhibiting the $\mathrm{A} \beta$ aggregation pathway from $\mathrm{A} \beta$ monomers to A11-positive oligomers and from A11-positive oligomers to $\mathrm{A} \beta$ deposition [42]. Although our study supports the reported findings above, our experiments were performed on a different $\mathrm{AD}$ rodent model which can completely mimic the neuropathological and behavioral alterations in $\mathrm{AD}$ by affecting several molecular changes associated with deprivation of endogenous estrogen and oxidative stress $[10,11]$. In addition to reduce $\mathrm{A} \beta$ accumulation, RA reversed mitochondrial swelling and crista deformations. Also, nucleus membrane integrity was maintained and normal perinuclear space was vied in the treatment group.

In a previous study, lipid peroxidation was detected before $\mathrm{A} \beta$ accumulation in 7 months $\mathrm{AD}$ transgenic mice [43]. In the current study, increase in brain TBARS and hippocampus 4-HNE levels in the OD group supports previous findings $[44,45]$ that show the importance of lipid peroxidation in the development of AD. In support of previous cell culture study [46], RA also decreased lipid peroxidation in our study. In addition, RA treatment attenuated 4-HNE level in the ODR rats. These findings suggest that RA could be used against $A \beta$ induced oxidative damage as a neuroprotective agent and also RA may prevent $\mathrm{A} \beta$ accumulation by decreasing lipid peroxidation. In addition, a significant increment in brain TBARS level was observed in the SH group. Since various routes of administration to animals like surgical operation, gavage and injection are known to be stressors, this data could be seen as quite reasonable. Although TBARS assay is sensitive and widely used method to measure the MDA level, TBARS level does not equal to lipid peroxidation and it is not specific to lipid peroxidation. Therefore, we also measured 4-HNE level to confirm TBARS test results. The same trend was not detected in the 4-HNE levels of sham operated and treated rats.

It was shown that suppression of the IL-1-mediated induction of COX-2/PGE2 pathway is one of the neuroprotective mechanism mediated by estrogen [47]. In addition, D-gal injection elevates COX-2 activity and expression in brain [48]. In parallel with these reports, COX-2 expression in the OD group was increased in comparison with $\mathrm{C}$ and $\mathrm{SH}$ groups in our study. This observation is also in agreement with previous findings [49] indicating the importance of this enzyme in 
the development of AD symptoms. On the other hand, the amount of PGE2 was found to be increased in the OD group compared with $\mathrm{C}$ and $\mathrm{SH}$ groups. Elevated level of PGE2 has been observed in the brains of $A D$ patients and mice $[8,9]$. In animal studies, PGE2 stimulates the production of $\mathrm{A} \beta$ by effecting $\beta$-secretase and $\beta$-secretase activity via some PGE2 receptors and promotes age-dependent oxidative damage $[8,50]$. Consistent with previous findings, our results also suggested that increased levels of this molecule may play a critical role in the accumulation of $\mathrm{A} \beta$ by triggering inflammatory mechanisms. Additionally, our findings support earlier reports suggesting that inhibition of COX-2 pathway and PGE2, is an important therapeutic target in $\mathrm{AD}$.

Anti-inflammatory effects of RA have been pointed in several studies [28, 51]. In a study on carcinogenesis, RA prevented the increase in PGE2 synthesis and eliminated COX-2 mRNA expression [28]. Additionally, repeated administration of RA remarkably reduced the arthritic index and COX-2 expression in synovial tissue in collagen induced arthritis [51]. The decrease of COX-2 expression and PGE2 levels in the ODR group compared with the OD group indicated that RA might be effective in suppressing the COX-2/ /PGE2 pathway. Consequently, RA might be beneficial in the treatment of $\mathrm{AD}$ by exerting anti-inflammatory effects and suppressing the synthesis of PGE2 in addition to its antioxidant properties.

One of the most important symptoms of $\mathrm{AD}$ is the impairment of cognition. As shown in previous studies $[10,11]$, increment in oxidative stress and estrogen deficiency, which are known to cause cognitive impairment, can mimic AD-like spatial memory dysfunction. Therefore, we performed the Y-maze test as a simple spatial recognition test which has been widely preferred to assess hippocampus-dependent short-term spatial memory [31]. We found that Y-maze performance was impaired in the OD group when the number of visits and the time spent were considered. Besides, the amount of time spent and the number of visits in the novel arm were markedly decreased in the OD group compared with the other groups. These results suggest that short-term spatial memory of OD rats was impaired.

In clinical trials, it was shown that Salvia officinalis which contains RA, improves cognitive functions of patients $[25,26]$. In accordance with this study, we found that RA significantly improved Y-maze performance in the ODR group. Additionally, various studies have shown that Alzheimer transgenic mice exhibit reduced locomotor activity in an open field test $[52,53]$. In a previous study [54], RA has been shown to increase the locomotor activity and motivation.
Consistent with these studies, beside short-term spatial memory, RA significantly increased the locomotor activity in the ODR group. It is important to note here that due to the increment in the locomotion, it can be assumed that increased novel arm frequency in the ODR group could be related to locomotion instead of improved short-term spatial memory. Although, the locomotion is a confounding factor for evaluation of Y-maze task performances, number of total arm entries was not different between the groups. Therefore, these findings suggest that treatment with RA may be a possible therapeutic approach for alleviating spatial learning and memory deficits in the AD rats. Taken together, RA may reverse some structural degeneration caused by early pathological events in AD including lipid peroxidation and activation of inflammatory pathways. To our knowledge this is the first study evaluating the effect of RA treatment on lipid peroxidation, COX-2/PGE2 pathway and cognitive alterations in that rat model of $\mathrm{AD}$. The other possible mechanisms underlying this beneficial effect remain to be determined, but may be at least partially due to decrease in oxidative stress and inflammatory responses.

\section{Conclusions}

In conclusion, our findings support that estrogen deficiency with increased oxidative stress may play an important role in the development and progression of $\mathrm{AD}$ and this model can be used to investigate the mechanisms underlying $\mathrm{AD}$ and new therapeutic strategies. Furthermore, RA treatment attenuates memory impairments through amelioration of oxidative stress and inflammatory response and is a potential candidate to slow the progression of the disease.

\section{Acknowledgements}

This study was supported by a grant from Akdeniz University Research Foundation, Turkey (2008.02.0122.004). This study was carried out as part of a MSc thesis by D. Kantar Gok presented to Akdeniz University Health Sciences Institute.

\section{References}

1. Maccioni RB, Munoz JP, Barbeito L. The molecular bases of Alzheimer's disease and other neurodegenerative disorders. Arch Med Res. 2001;32:367-381. doi: 10.1016/S01884409(01)00316-2.

2. Pratico D, MY Lee V, Trojanowski JQ, Rokach J, Fitzgerald GA. Increased F2-isoprostanes in Alzheimer's disease: evidence for enhanced lipid peroxidation in vivo. FASEB J. 1998;12:1777-1783. PMID: 9837868.

3. Schuessel K, Schafer S, Bayer TA et al. Impaired $\mathrm{Cu} / \mathrm{Zn}-\mathrm{SOD}$ activity contributes to increased oxidative damage in APP 
transgenic mice. Neurobiol Dis. 2005;18:89-99. doi: 10.1016/j. nbd.2004.09.003.

4. David DC, Hauptmann S, Scherping I et al. Proteomic and functional analyses reveal a mitochondrial dysfunction in P301L tau transgenic mice. J Biol Chem. 2005;280:23802-23814. doi: 10.1074/jbc.M500356200.

5. Smith CD, Carney JM, Starke-Reed PE et al. Excess brain protein oxidation and enzyme dysfunction in normal aging and in Alzheimer disease. Proc Natl Acad Sci USA. 1991;188:10540-10543. PMID: 1683703.

6. Bazan NG, Colangelo V, Lukiw WJ. Prostaglandins and other lipid mediators in Alzheimer's disease. Prostaglandins Lipid Mediat. 2002;69:197-210. PMID: 12432919.

7. Minghetti L. Role of COX-2 in inflammatory and degenerative brain diseases. Subcell Biochem. 2007;42:127-141. doi: 10.1080/07391102.2013.839960.

8. Liang $\mathrm{X}$, Wang Q, Hand $\mathrm{T}$ et al. Deletion of the prostaglandin E2 EP2 receptor reduces oxidative damage and amyloid burden in a model of Alzheimer's disease. J Neurosci. 2005;25:10180-10187. doi: 10.1523/JNEUROSCI.3591-05.2005.

9. Montine TJ, Sidell KR, Crews BC et al. Elevated CSF prostaglandin E2 levels in patients with probable AD. Neurology. 1999;53:1495-1498. doi: 10.1212/WNL.55.2.323.

10. Hua X, Lei M, Zhang Y et al. Long-term D-galactose injection combined with ovariectomy serves as a new rodent model for Alzheimer's disease. Life Sci. 2007;80:1897-1905. doi: 10.1016/j.bbrc.2015.04.031.

11. Zhang X, Wang J, Xing Y et al. Effects of ginsenoside Rg1 or $17 \beta$-estradiol on a cognitively impaired, ovariectomized rat model of Alzheimer's disease. Neuroscience. 2012;220:191-200. doi: 10.1016/j.neuroscience.2012.06.027.

12. Gibbs RB. Impairment of basal forebrain cholinergic neurons associated with aging and long-term loss of ovarian function. Exp Neurol. 1998;151:289-302. doi: 10.1006/exnr.1998.6789.

13. Paganini-Hill A, Henderson VW. Estrogen deficiency and risk of Alzheimer's disease in women. Am J Epidemiol. 1994;140:256-261. doi: 10.1016/0378-5122(95)93906-A.

14. Good PF, Werner P, Hsu A, Olanow CW, Perl DP. Evidence for neuronal oxidative damage in Alzheimer's disease. Am J Pathol. 1996;149:21-28. PMID: 8686745.

15. Schippling S, Kontush A, Arlt S et al. Increased lipoprotein oxidation in Alzheimer's disease. Free Radical Biol Med. 2000;28:351-360. doi: 10.1016/S0891-5849(99)00247-6.

16. Green PS, Simpkins JW. Neuroprotective effects of estrogens: potential mechanisms of action. Int J Dev Neurosci. 2000;18:347-358. doi: 10.1016/S0736-5748(00)00017-4.

17. Persson I, Yuen J, Bergkvist L, Schairer C. Cancer incidence and mortality in women receiving estrogen and estrogen-progestin replacement therapy long-term follow-up of a Swedish cohort. Int J Cancer. 1996;67:327-332. doi: 10.1002/(SICI)1097-0215(19960729)67:3<327::AIDIJC4>3.0.CO;2-T.

18. Steinberg KK, Thacker SB, Smith SJ et al. A meta-analysis of the effect of estrogen replacement therapy on the risk of breast cancer. JAMA. 1991;265:1985-1990. doi: 10.1001/ jama.1991.03460150089030.

19. Imanshahidi $M$, Hosseinzadeh $H$. The pharmacological effects of salvia species on the central nervous system. Phytother Res. 2006;20:427-437. doi: 10.1002/ptr.1898.

20. Mantle D, Pickering AT, Perry EK. Medicinal plant extracts for the treatment of dementia: a review of their pharmacology, efficacy and tolerability. CNS Drugs. 2000;13:201-213.

21. Tildesley NT, Kennedy DO, Perry EK et al. Salvia lavandulaefolia (Spanish sage) enhances memory in healthy young volunteers. Pharmacol Biochem Behav. 2003;75:669-674. doi: 10.1016/S0091-3057(03)00122-9.

22. Tildesley NT, Kennedy DO, Perry EK, Ballard CG, Wesnes KA, Scholey AB. Positive modulation of mood and cognitive performance following administration of acute doses of Salvia lavandulaefolia essential oil to healthy young volunteers. Physiol Behav. 2005;83:699-709. doi: 10.1016/j.physbeh.2004.09.010.

23. KennedyDO,PaceS,HaskellC, OkelloEJ, MilneA,ScholeyAB. Effects of cholinesterase inhibiting sage (Salvia officinalis) on mood, anxiety and performance on a psychological stressor battery. Neuropsychopharmacology. 2006;31:845-852. doi: 10.1038/sj.npp.1300907.

24. Scholey AB, Tildesley NT, Ballard CG et al. An extract of Salvia (sage) with anticholinesterase properties improves memory and attention in healthy older volunteers. Psychopharmacology. 2008;198:127-139. doi: 10.1007/s00213-008-1101-3.

25. Akhondzadeh S, Noroozian M, Mohammadi M, Ohadinia S, Jamshidi AH, Khani M. Salvia officinalis extract in the treatment of patients with mild to moderate Alzheimer's disease: A double blind, randomized and placebo-controlled trial. J Clin Pharm Ther. 2003;28:53-59. doi: 10.1046/j.13652710.2003.00463.x.

26. Perry NSL, Bollen C, Perry EK, Ballard C. Salvia for dementia therapy: review of pharmacological activity and pilot tolerability clinical trial. Pharmacol Biochem Behav. 2003;75:651-659. doi: 10.1016/S0091-3057(03)00108-4.

27. Petersena M, Simmonds MSJ. Rosmarinic Acid. Phytochemistry. 2003;62:121-125. doi: 10.1016/S0037-6337(09)70275-X.

28. Osakabe N, Yasuda A, Natsume M, Yoshikawa T. Rosmarinic acid inhibits epidermal inflammatory responses: anticarcinogenic effect of Perilla frutescens extract in the murine two-stage skin model. Carcinogenesis. 2004;25:549-557. doi: 10.1093/carcin/bgh034.

29. Hohmann J, Zupkó I, Rédei D et al. Protective effects of the aerial parts of Salvia officinalis, Melissa Officinalis and Lavandula angustifolia and their constituents against enzyme-dependent and enzyme-independent lipid peroxidation. Planta Med. 1999;65:576-578. doi: 10.1055/s-2006-960830.

30. Perry EK, Pickering AT, Wang WW, Houghton PJ, Perry NS. Medicinal plants and Alzheimer's disease: from ethnobotany to phytotherapy. J Pharm Pharmacol. 1999;51:527-534. doi: 10.1016/j.jep.2007.11.015.

31. Tanriover G, Sozen B, Seker A, Kilic T, Gunel M, Demir N. Ultrastructural analysis of vascular features in cerebral cavernous malformations. Clin Neurol Neurosurg. 2013;115:438-444. doi: 10.1016/j.clineuro.2012.06.023.

32. Wasowicz W, Jean N, Peratz A. Optimized steps in fluorometric determination of thiobarbituric acid-reactive substances in serum. Importance of extraction $\mathrm{pH}$ and influence of sample, preservation and storage. Clin Chem. 1993;39:2522-2526. PMID: 8252725.

33. Aslan M, Yucel I, Ciftcioglu A, Savas B, Akar Y, Yucel G. Corneal protein nitration in experimental uveitis. Exp Biol Med. 2007;232:1308-1313. doi: 10.3181/0702-RM-34.

34. Dellu F, Fauchey V, Le Moal M, Simon H. Extension of a new two-trial memory task in the rat: influence of environmental context on recognition processes. Neurobiol Learn Mem. 1997;67:112-120. doi: 10.1006/nlme.1997.3746.

35. Jamshed N, Ozair FF, Aggarwal P, Ekka M. Alzheimer disease in post-menopausal women: Intervene in the critical window period. Neurology. 2000;54:833-837. doi: 10.4103/0976-7800.127791.

36. Rasgon NL, Magnusson C, Johansson ALV, Pedersen NL, Elman S, Gatz M. Endogenous and exogenous hormone expo- 
sure and risk of cognitive impairment in Swedish twins: a preliminary study. Psychoneuroendocrinology. 2005;30:558-567. doi: 10.1016/j.psyneuen.2005.01.004.

37. Sobow T, Kloszewska I. Modulation of age at onset in late-onset sporadic Alzheimer's disease by estrogen-related factors: the age of menopause and number of pregnancies. German J Psychiatry. 2003;6:49-55.

38. Cui X, Zuo P, Zhang Q et al. Chronic systemic D-galactose exposure induces memory loss, neurodegeneration, and oxidative damage in mice: protective effects of R-alpha-lipoic acid.J Neurosci Res. 2006;84:647-654. doi: 10.1002/jnr.20899.

39. Zhao HF, Li Q, Li Y. Long-term ginsenoside administration prevents memory loss in aged female C57BL/6J mice by modulating the redox status and up-regulating the plasticity-related proteins in hippocampus. Neuroscience. 2011;183:189-202. doi: 10.1016/j.neuroscience.2011.03.048.

40. Ohkura T, Isse K, Akazawa K, Hamamoto M, Yaoi Y,HaginoN. Evaluation of estrogen treatment in female patients with dementia of the Alzheimer type. Endocr J. 1994;41:361-371. doi: $10.1507 /$ endocrj.41.361.

41. Ono K, Li L, Takamura Y et al. Phenolic compounds prevent amyloid $\beta$-protein oligomerization and synaptic dysfunction by site-specific binding. J Biol Chem. 2012;287:14631-1443. doi: 10.1074/jbc.M111.325456.

42. Hamaguchi T, Ono K, Murase A, Yamada M. Phenolic compounds prevent Alzheimer's pathology through different effects on the amyloid-beta aggregation pathway. Am J Pathol. 2009;175:2557-2565. doi: 10.2353/ajpath.2009.090417.

43. Pratico D, Uryu K, Leight S, Trojanoswki JQ, Lee VM. Increased lipid peroxidation precedes amyloid plaque formation in an animal model of Alzheimer amyloidosis. J Neurosci. 2001;21:4183-4187. PMID: 11404403.

44. Butterfield DA, Reed T, Perluigi M et al. Elevated protein-bound levels of the lipid peroxidation product, 4-hydroxy-2-nonenal, in brain from persons with mild cognitive impairment. Neurosci Lett. 2006;397:170-173. doi: 10.1016/j. neulet.2005.12.017.

45. Williams TI, Lynn BC, Markesbery WR, Lovell MA. Increased levels of 4-hydroxynonenal and acrolein, neurotoxic markers of lipid peroxidation, in the brain in Mild
Cognitive Impairment and early Alzheimer's disease. Neurobiol Aging. 2006;27:1094-1099. doi: 10.1016/j.neurobiolaging.2005.06.004.

46. Iuvone T, Filippis D, Esposito G, D'Amico A, Izzo A. The spice sage and its active ingredient Rosmarinic acid protect PC1 2 cells from amyloid- $\beta$ peptide-induced neurotoxicity. J Pharmacol Exp Ther. 2006;317:1143-1149. doi: 10.1124/ jpet.105.099317.

47. Ospina JA, Brevig HN, Krause DN, Duckles SP. Estrogen suppresses IL-1beta-mediated induction of COX-2 pathway in rat cerebral blood vessels. Am J Physiol. 2004;286:2010-2019. doi: 10.1152/ajpheart.00481.2003.

48. Tsai SJ, Yin MC. Anti-glycative and anti-inflammatory effects of protocatechuic acid in brain of mice treated by D-galactose. Food Chem Toxicol. 2012;50:3198-3205. doi: 10.1016/j. fct.2012.05.056.

49. Hoozemans JJ, O'Banion MK. The Role of COX-1 and COX-2 in Alzheimer's disease pathology and the therapeutic potentials of non-steroidal anti-inflammatory drugs. Curr Drug Targets CNS Neurol Disord. 2005;4:307-315. doi: 10.2174/1568007054038201.

50. Hoshino T, Nakaya T, Homan T et al. Involvement of prostaglandin E2 in production of amyloid- $\beta$ peptides both in vitro and in vivo. $J$ Biol Chem. 2007;282:32676-32688. doi: 10.1016/j.jalz.2010.05.1365.

51. Youn J, Lee KH, Won J et al. Beneficial effects of rosmarinic acid on suppression of collagen induced arthritis.J Rheumatol. 2003;30:1203-1207. PMID: 12784390.

52. Gortz N, Lewejohanna L, Tomma M et al. Effects of environmental enrichment on exploration, anxiety, and memory in female TgCRND8 Alzheimer mice. Behav Brain Res. 2008;191:43-48. doi: 10.1016/j.bbr.2008.03.006.

53. King DL, Arendash GW. Behavioral characterization of the Tg2576 transgenic model of Alzheimer's disease through 19 months. Physiol Behav. 2002;75:627-642. doi: 10.1016/ S0031-9384(02)00639-X

54. Takeda H, Tsuji M, Inazu M, Egashira T, Matsumiya T. Rosmarinic acid and caffeic acid produce antidepressive-like effect in the forced swimming test in mice. Eur J Pharmacol. 2002;449:261-267. doi: 10.1016/S0014-2999(02)02037-X.

Submitted: 9 July, 2015

Accepted after reviews: 17 December, 2015 Available as AoP: 21 December, 2015 